\begin{tabular}{|c|}
\hline \\
Original \\
Article
\end{tabular}

\title{
What is normal vaginal flora?
}

\author{
C J F Priestley, B M Jones, J Dhar, Linda Goodwin
}

Objective: To observe the composition of the vaginal flora of healthy women over time, and in relation to hormonal changes, sexual activity, and hygiene habits.

Design: A longitudinal surveillance of the vaginal flora over an eight week period.

Subjects: 26 female health care workers in local genitourinary medicine clinics.

Methods: The participants were anonymised. They filled in diary cards daily. Blind vaginal swabs were self-taken two-seven times weekly. A smear was air-dried for later Gram staining. The swabs were also cultured for Candida spp, Gardnerella vaginalis, anaerobes, Mycoplasma hominis and Ureaplasma urealyticum.

Results: Of 26 subjects, only four had normal vaginal microbiology throughout. One woman, who was not sexually active, had bacterial vaginosis (BV) throughout and nine (35\%) had intermittent BV. Candidiasis was found intermittently in eight women (31\%), and eight had normal microscopy. U urealyticum was isolated intermittently in $40 \%$ of women with $\mathrm{BV}, 25 \%$ with candida, and $50 \%$ with normal microscopy. Many women were symptomatic, but symptoms correlated poorly with microbiological findings. All but two women were sexually active; however, more women with BV were exposed to semen. BV seemed to be related to frequent use of scented soap, and there appeared to be an additive effect of clothing and hygiene factors.

Conclusions: Our study raises doubts about what should be regarded as normal vaginal flora. It calls into question the significance of finding BV or $U$ urealyticum on a single occasion in asymptomatic women, or of finding normal flora in symptomatic women. The effect of external factors on the vaginal flora deserve further study.

(Genitourin Med 1997;73:23-28)

Keywords: vaginal flora; bacterial vaginosis; candidiasis; ureaplasma

\section{Introduction}

Bacterial vaginosis was first reported in 1955 by Gardner and Dukes, ${ }^{1}$ who described the unique clinical signs and symptoms and the distinctive nature of the vaginal discharge associated with it. They also described a "new" causative organism, which they named "Haemophilis vaginalis", subsequently renamed Gardnerella vaginalis.

Forty years on, while the complexity of the microbiological picture has been well described, ${ }^{23}$ the aetiology and pathogenesis of the condition remain poorly understood, and the reasons as to why the normal, healthy lactobacillus flora is superseded by an overwhelming "cocktail" of bacteria are still not known. Fortunately, laboratory diagnosis can be readily made by simple, Gram stain microscopy where "clue cells" and the typical, abnormal bacterial flora of BV are easily recognised. ${ }^{4}$

The experience of some workers suggests that factors such as hormonal status, coitus, and exposure to semen may play an important role in the pathogenesis of BV. We were also interested in the role of clothing and hygiene habits such as the use of certain bath additives, which are said to precipitate vulvovaginal candidiasis in some women. As there are few documented studies on these aspects, we designed a longitudinal study to observe the composition of the vaginal flora in healthy women, in relation to hormonal changes and external factors.

\section{Methods \\ Volunteer women}

The study took place over an eight week period to cover two menstrual cycles. Subjects were recruited from health care staff of genitourinary medicine departments in Sheffield, Rotherham, and Barnsley. They were anonymised to encourage participation and increase the accuracy of recorded personal data. Application was made to the local Ethics Committee; however it was felt that formal approval for the study was not required as participation was both voluntary and anonymous. The participants randomly chose numbered packs containing swabs, slides and Amies transport media, and were asked to complete diary forms to record age, contraceptive method used, menstruation, tampon use, coitus, apparel (tights and trousers), bath additives, and any symptoms of discharge, irritation, or odour. The diary forms were retained separately until the end of the study.

The women took blind vaginal swabs from themselves two-seven times per week, prior to bathing. They prepared air-dried smears, for subsequent Gram staining, before placing the swab into the transport medium for culture. These swabs were left at room temperature until the participant could deliver them to the clinic, and swabs taken on a Friday or Saturday, in particular, could be left for two to three days before being plated out. For this reason we placed greater emphasis on the 
Gram stained slides than cultures, in particular for the diagnosis of BV. The samples, identified only by number, were tested "blind" in the laboratory. We also asked the participants to measure the $\mathrm{pH}$ of the secretions, using standard paper strips, prior to preparing the slides; however, we became concerned about the accuracy of a reading from a blind vaginal swab because of potential contamination from the vulva or cervical mucosa, and $\mathrm{pH}$ was not measured by all the participants.

\section{Microbiological tests}

Microscopy Gram stained smears were examined for the presence of lactobacilli, "clue cells", "gram-variable" organisms, curved rods and yeasts. The results were recorded as "normal", where the flora comprised lactobacilli only; "normal, but with yeasts"; "intermediate", with an abnormal BV-type flora but some lactobacilli still present; or "bacterial vaginosis", where the smears showed "clue cells" and masses of gram-variable bacteria, and a complete absence of lactobacilli. ${ }^{4}$

Cultures The swabs were cultured for $G$ vaginalis, anaerobic organisms, Mycoplasma hominis, Ureaplasma urealyticum, and yeasts. Plates of Columbia agar (Unipath Ltd, Basingstoke, UK) containing $7 \%$ horse blood were inoculated and incubated, at $37^{\circ} \mathrm{C}$ in an atmosphere of $5 \% \mathrm{CO}_{2}$ in air for 48 hours. $G$ vaginalis isolates were identified by Gram stain and hippurate hydrolysis tests. Yeasts were confirmed by microscopy only but were assumed to be Candida spp. For the isolation of anaerobic organisms, plates of Columbia Blood agar, containing kanamycin at $75 \mu \mathrm{g}$ per $\mathrm{ml}$ were inoculated and incubated for 48 hours, at $37^{\circ} \mathrm{C}$ in an anaerobic cabinet (Don Whitley Scientific Ltd, UK) containing nitrogen $80 \%$, hydrogen $10 \%$ and carbon dioxide $10 \%$. A $50 \mu \mathrm{g}$ metronidazole disc was added to the inoculated streaks and, if a zone of colony inhibition was seen after incubation, the presence of "anaerobes" was recorded. Cultures for the isolation of mycoplasma and Ureaplasma spp were made by inoculating plates of "MUD" medium (Unipath Ltd, Basingstoke, UK) directly with the swab, and incubating for two days in the anaerobic cabinet. Cultures were examined and species identified using a $\times 10$ microscope objective.

Analysis Statistical analysis was performed using Epi info, version 6. Results were analysed using the Fisher exact 2-tailed test for small numbers and the chi square test for larger numbers.

\section{Results}

All female staff working in the three genitourinary medicine clinics (approximately 45 women) were invited to participate and 33 were entered into the study. However, four women did not complete the study and three did not return their diaries. Of the remaining 26 women, three took fewer than two swabs per week overall $(1 \cdot 7,1 \cdot 8,1 \cdot 9$ per week $)$ and a further three only completed six or seven weeks of the study, but we felt that sufficient data were obtained to include them in the analysis. Overall, the 26 women included in the analysis took a mean of 3.2 swabs per week (range $1 \cdot 7-7 \cdot 0$ ) for a six to eight week period.

\section{Microbiology}

One woman had BV throughout the period of study. $G$ vaginalis, anaerobes, Ureaplasma urealyticum and Mycoplasma hominis were isolated from the majority of her swabs. She complained of some irritation but not of vaginal discharge or odour, the typical symptoms of BV. BV was found intermittently in nine women $(34.6 \%)$ in between 10 and $62 \%$ (mean $27.5 \%$ ) of swabs taken. $G$ vaginalis was isolated from $64 \%$ and anaerobes from $28 \%$ of swabs showing BV.

Candida $s p p$ were found intermittently in eight women (30.8\%) in between 14 and $70 \%$ (mean $35.5 \%$ ) of swabs taken. Only eight women $(30 \cdot 8 \%)$ had normal microscopy throughout.

Ureaplasma urealyticum was isolated intermittently in $10(38.5 \%)$ women; four $(40 \%)$ of 은 those with BV, two (25\%) of those with can- didiasis, and four (50\%) of those with normal T microscopy. Table 1 shows the characteristics of these women. The presence of $U$ urealyticum did not appear to be related to sexual activity or exposure to semen.

Mycoplasma hominis was only isolated in two women - the one with constant BV, in whom it was isolated in $96 \%$ of swabs, and one other with intermittent BV, in whom it was found in $3 \%$ of swabs taken. $\beta$-haemolytic streptococci were isolated from two women, both of whom had Candida spp; in one it was found only on a single occasion, and in the other it was isolated from four consecutive swabs ( $22 \%$ of total) during the second week of the study.

Overall, only four out of 26 , or $15 \%$ of women, had apparently completely normal flora throughout.

There were no significant differences in the number of swabs taken per week between the women with BV, Candida spp, normal microscopy, or $U$ urealyticum, and in addition, there were no significant differences in the $\tilde{\sigma}$ proportions of positive swabs taken on a $N$ Friday or Saturday with those taken the rest of N the week. This suggests that the intermittent nature of the microbiological findings was not 0 due to a significant loss of sensitivity arising from the culture methods.

Table 1 Characteristics of women with $U$ urealyticum (UU) isolated from vaginal swabs

\begin{tabular}{llcl}
\hline Subject no & Microscopy & $\begin{array}{l}\text { UU-\% of } \\
\text { swabs taken }\end{array}$ & $\begin{array}{l}\text { Exposure to } \\
\text { semen }\end{array}$ \\
\hline 5 & normal & $6 \cdot 5$ & + \\
6 & normal & $7 \cdot 7$ & + \\
7 & normal & $53 \cdot 8$ & - \\
8 & normal & $73 \cdot 3$ & - \\
9 & candida & $81 \cdot 0$ & + \\
16 & candida & $2 \cdot 7$ & - \\
20 & BV & $20 \cdot 0$ & + \\
22 & BV & $74 \cdot 1$ & + \\
23 & BV & $82 \cdot 4$ & + \\
26 & BV & $89 \cdot 3$ & - \\
\hline
\end{tabular}

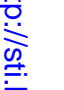


Table 2 Characteristics of women with BV, candidiasis, and normal microscopy

\begin{tabular}{|c|c|c|c|c|}
\hline Variable & $\begin{array}{l}\text { Normal } \\
(n=8)\end{array}$ & $\begin{array}{l}\text { Candida } \\
(n=8)\end{array}$ & $\begin{array}{l}B V \\
(n=10)\end{array}$ & $\begin{array}{l}p \text { value } \\
B V \text { vs normal }\end{array}$ \\
\hline Average age range & $39 \cdot 4$ & $35 \cdot 6$ & $40 \cdot 5$ & - \\
\hline Sexually active & 8 & 7 & 9 & 0.91 \\
\hline $\begin{array}{l}\text { Coitus per week } \\
\text { (range) }\end{array}$ & $\begin{array}{l}2 \cdot 1 \\
(0 \cdot 6-4 \cdot 5)\end{array}$ & $\begin{array}{c}1 \cdot 5 \\
(0-2 \cdot 4)\end{array}$ & $\begin{array}{l}1 \cdot 4 \\
(0-2 \cdot 5)\end{array}$ & - \\
\hline Exposure to semen & $5(62.5 \%)$ & $4(50 \%)$ & $\begin{array}{l}(0-2 \cdot 3) \\
9(90 \%)\end{array}$ & $0 \cdot 27$ \\
\hline Menstruating & 7 & 7 & 9 & 1.00 \\
\hline Tampon use & $6(75 \%)$ & $7(88 \%)$ & $6(60 \%)$ & 0.64 \\
\hline Tampons $>50 \%$ of menstruation & $4(50 \%)$ & $7(88 \%)$ & $4(40 \%)$ & 1.00 \\
\hline \multicolumn{5}{|l|}{ Symptoms: } \\
\hline Discharge $>2$ days per week & $1(13 \%)$ & $1(13 \%)$ & $1(10 \%)$ & $1 \cdot 00$ \\
\hline Irritation $>2$ days per week & $0(0 \%)$ & $1(13 \%)$ & $1(10 \%)$ & $1 \cdot 00$ \\
\hline Odour $>2$ days per week & $0(0 \%)$ & $0(0 \%)$ & $1(10 \%)$ & 1.00 \\
\hline \multicolumn{5}{|l|}{ External factors: } \\
\hline Tights $>2$ days per week & $4(50 \%)$ & $6(75 \%)$ & $6(60 \%)$ & $1 \cdot 00$ \\
\hline Trousers $>2$ days per week & $8(100 \%)$ & $5(63 \%)$ & $7(70 \%)$ & $0 \cdot 22$ \\
\hline Bubble bath $>2$ days per week & $2(25 \%)$ & $4(50 \%)$ & $4(40 \%)$ & 0.64 \\
\hline Scented soap $>2$ days per week & $2(25 \%)$ & $4(50 \%)$ & $9(90 \%)$ & $0 \cdot 009^{\star}$ \\
\hline$>3$ of above $>2$ days per week & $1(13 \%)$ & $3(38 \%)$ & $7(70 \%)$ & $0 \cdot 02^{\star}$ \\
\hline
\end{tabular}

There were no significant differences in these variables between the BV and candida groups, or between those with candida and normal microscopy.

The women were divided for analysis into three groups; those with normal flora, candidiasis, and BV on microscopy. There was no significant difference in the age groups of the women.

\section{Effect of sexual activity}

All but two were sexually active (table 2). Those with normal flora tended to have intercourse more frequently, but there was no significant difference between the groups. However, all nine women with intermittent BV had unprotected sex, compared with $50 \%$ of those with candidiasis and $63 \%$ of those with normal microscopy, although this difference did not reach significance $(p=0 \cdot 2)$. Details of oral sexual contact were not obtained. The woman with constant BV had never had sex with a man, and had not been sexually active at all for the previous five years. The numbers were too small to draw any conclusions about the effects of contraception.

\section{Effect of menstruation}

Two women had had hysterectomies, and one was post-menopausal. For the remaining women, there was no difference between the three groups in the duration of menstruation, but the women with candidiasis appeared to use tampons for a greater proportion of this time (table 2). A comparison of the weeks of the menstrual cycle during which $\mathrm{BV}$ and Candida spp were present showed that BV was more common during the first week of the cycle, whereas Candida spp were found more frequently during the last week (table 3 ).

\section{Relation with symptoms}

There was no significant difference between the groups in the numbers of women with discharge, odour, or irritation for more than two days per week (table 2).

We looked at symptoms in the individual subjects (fig 1). In these graphs, the subjects are ranged in ascending order of the frequency which abnormal swabs were found, and there was no correlation between the frequency with which BV, Candida spp, or $U$ urealyticum were found and the frequency of symptoms. Subject 25 , with intermittent $\mathrm{BV}$, appears to be symptomatic, but she actually complained of symptoms on as many days when the microbiology was normal as when it was abnormal. The same was true for women with candidiasis, although three women (subjects 9,13 , and 15) were symptomatic enough to treat themselves. The women with normal microscopy had fewer symptoms overall, with one exception. Subject 6, with normal microscopy, had $U$ urealyticum isolated from $8 \%$ of swabs taken; its presence was not related to symptoms. There was no correlation between the symptoms and the menstrual cycle.
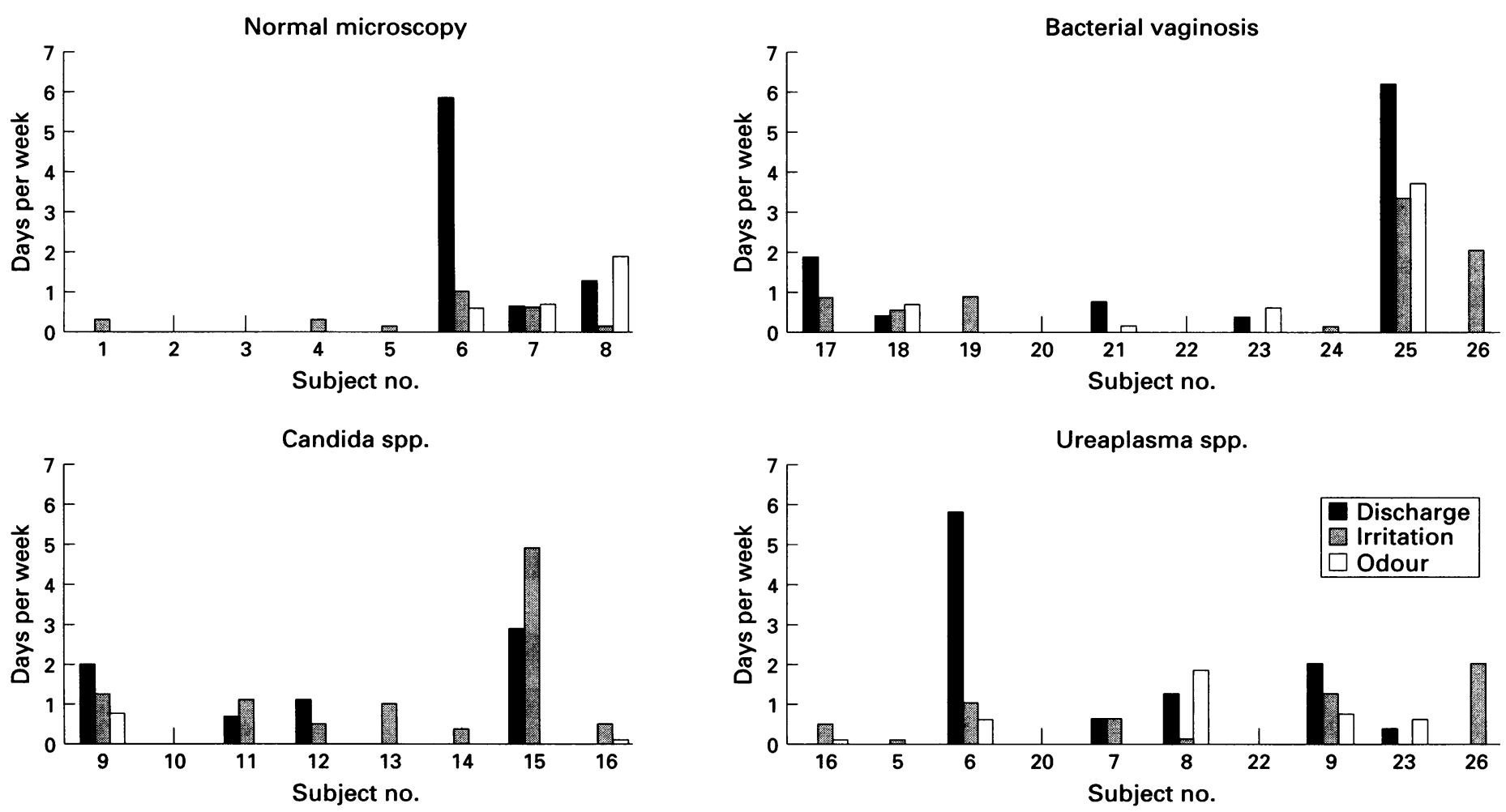

Figure 1 Symptoms in relation to microbiology of the vagina. 
Figure 2 Longitudinal changes in flora in Subject 23.
Subject no. 23

Contraception: coil

Age: $35-39$ years
Normal

Abnormal

Intermediate

Bacterial vaginosis

Spermatozoa

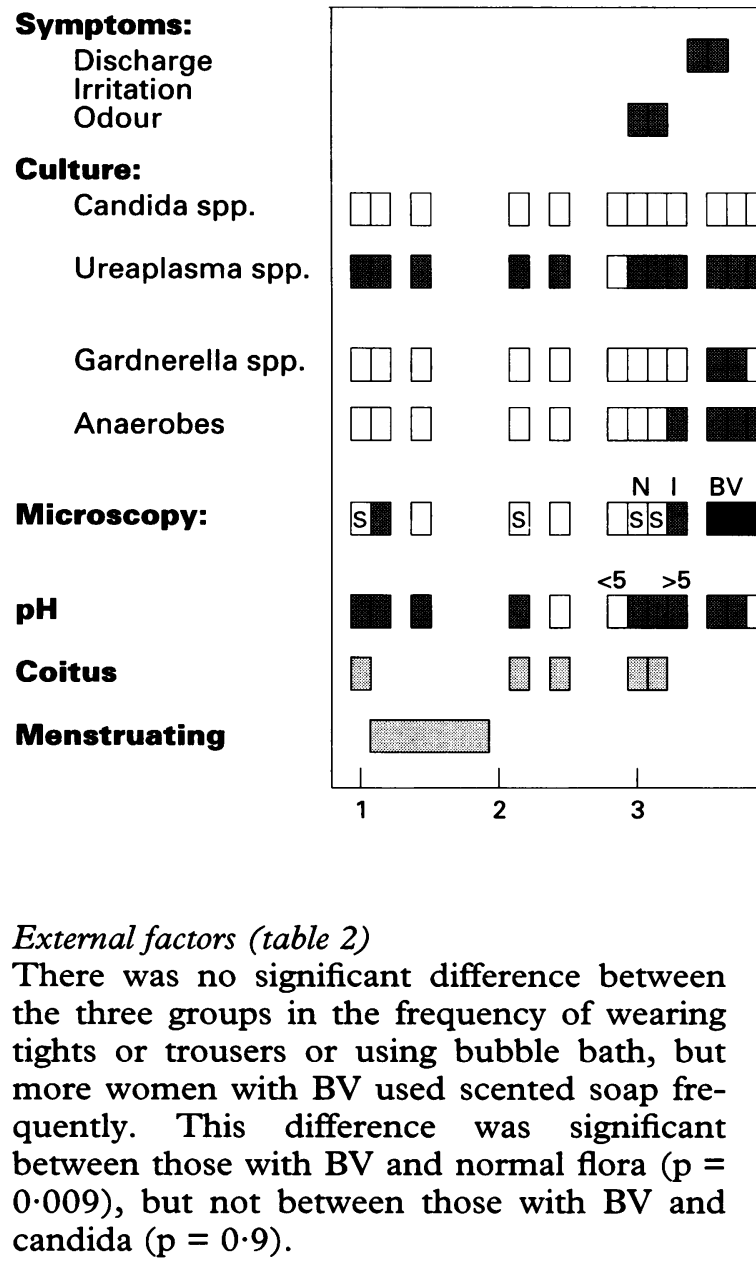

Figure 3 Longitudinal changes in vaginal flora in Subject 18.
Subject no. 18

Contraception: POP

Age: $\mathbf{3 0 - 3 4}$ years
There also appeared to be an additive effect; women with BV were significantly more likely $\overrightarrow{0}$ to use three or more of these factors for more $\stackrel{\bullet}{-}$ than two days a week ( $p=0.03)$.

We have encountered patients with BV who are overzealous with washing because they are aware of an odour. To determine if this could

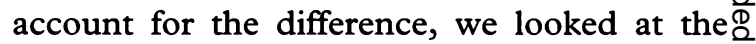
relationship between odour and the use of $\overrightarrow{\vec{O}}$

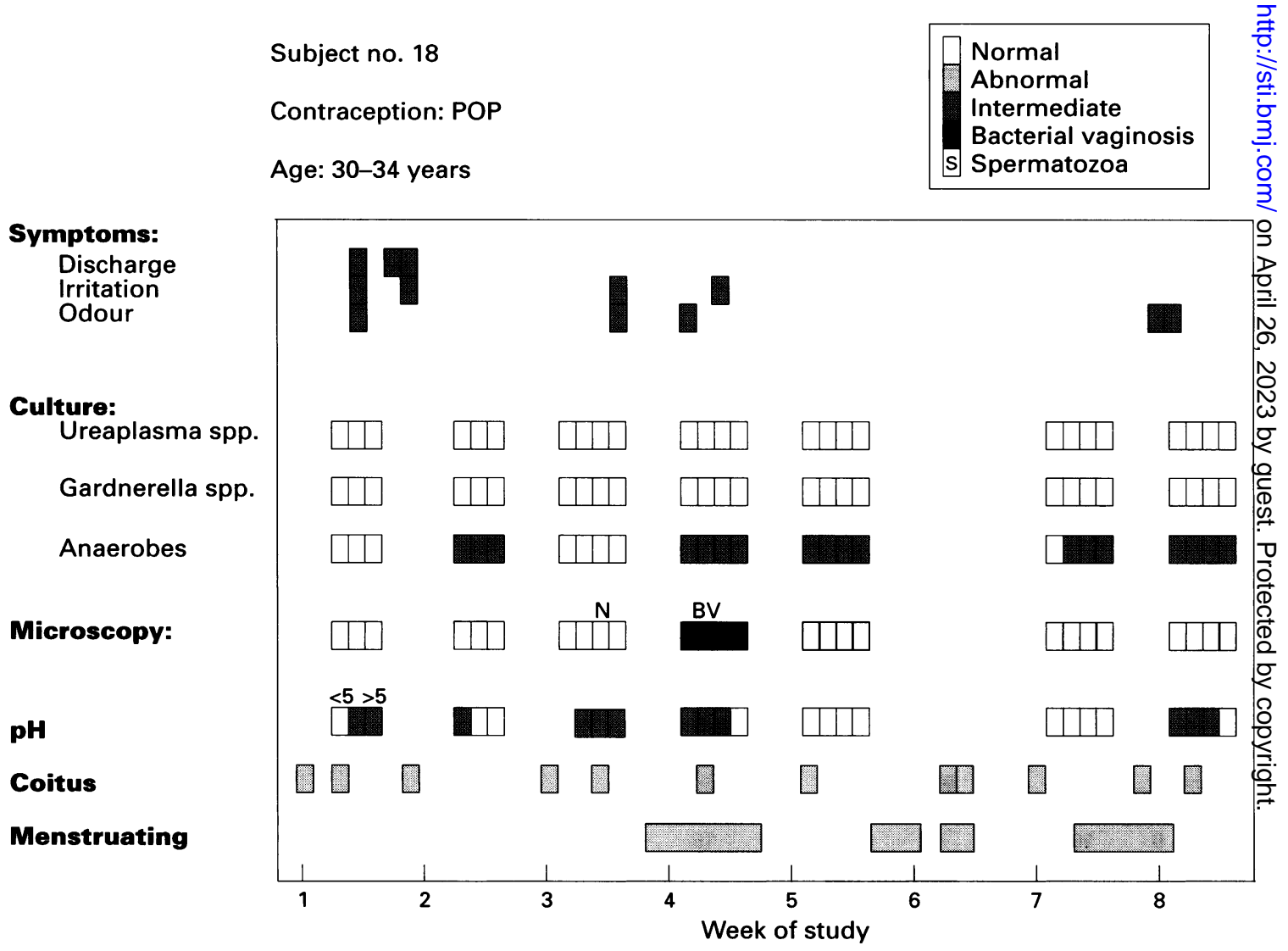




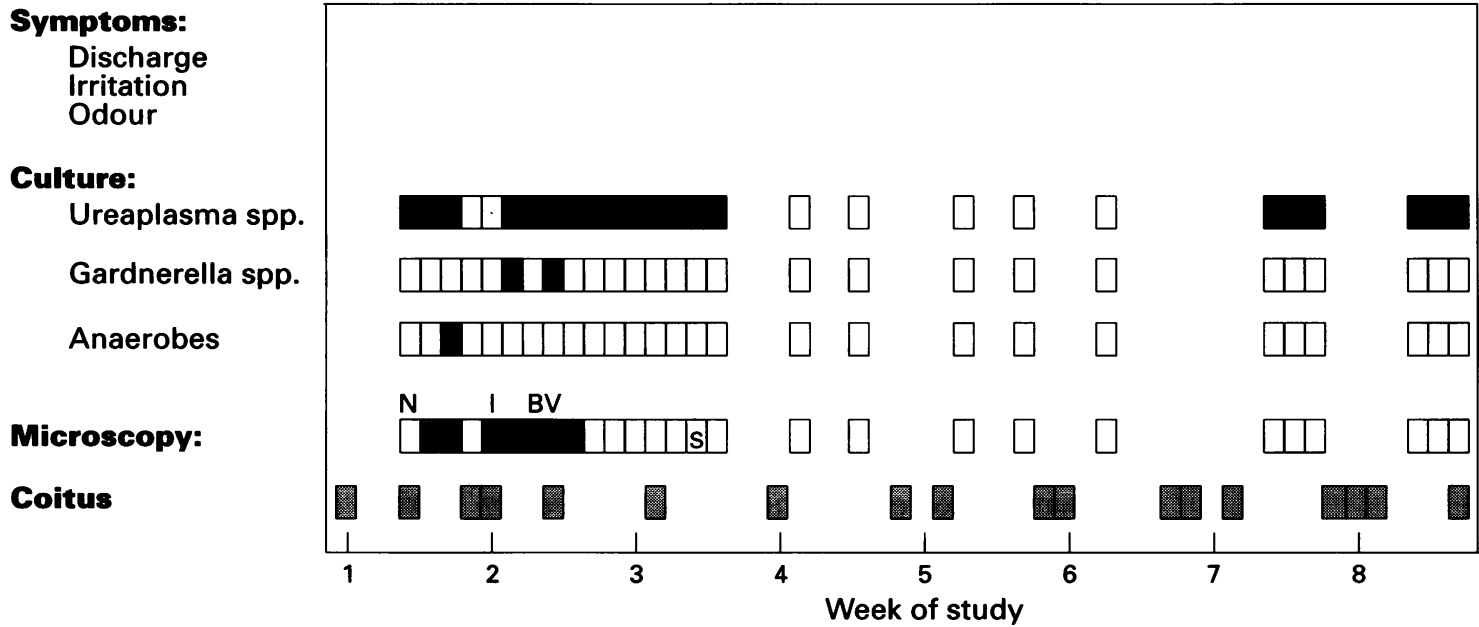

scented soap. There was no correlation, suggesting that in our cohort, more frequent use of scented soap was not due to increased awareness of odour.

\section{Longitudinal changes in women with intermittent $B V$}

The microbiological findings of the women with intermittent BV, together with data from their diaries, were looked at in detail. Subject 23 (fig 2) had BV or intermediate flora on $32 \%$ of swabs taken. Intermediate flora was noted on four occasions, and on two of these it progressed to BV. This appeared to occur in the second half of the menstrual cycle, following periods of frequent sexual activity. The appearances of BV correlated with the presence of $G$ vaginalis and anaerobes on culture. $U$ urealyticum was present on the majority of swabs taken. The $\mathrm{pH}$ rose, as would be expected, during menstruation, and also after the periods of frequent coitus. It was noted that the periods when BV was found were preceded by a rise in $\mathrm{pH}$. This woman had few symptoms, which were not related to abnormalities in the flora.

Subject 18 (fig 3) had BV during one week of the study ( $15 \%$ of swabs taken). Anaerobes were isolated during this period and also frequently when the microscopy was normal. Again, the period of BV was preceded by a rise in $\mathrm{pH}$. Her few symptoms were not correlated to the vaginal flora.

Subject 22 (fig 4) was post-menopausal; she had two episodes of $\mathrm{BV}$ at the beginning of the

Table 3 Effect of menstrual cycle on the vaginal flora (menstruating women with BV or Candidiasis; $n=16$ )

\begin{tabular}{|c|c|c|c|c|c|c|}
\hline \multirow[b]{2}{*}{$\begin{array}{l}\text { Days of } \\
\text { menstrual cycle }\end{array}$} & \multicolumn{2}{|c|}{ Candidiasis $(n=7)$} & \multirow[b]{2}{*}{$p$ value } & \multicolumn{2}{|c|}{ Total $B V(n=9)$} & \multirow[b]{2}{*}{$p$ value } \\
\hline & $\begin{array}{l}\text { total swabs } \\
\text { taken }\end{array}$ & $\%$ positive & & $\begin{array}{l}\text { total swabs } \\
\text { taken }\end{array}$ & $\%$ positive & \\
\hline $\begin{array}{l}1 \text { to } 7 \\
8 \text { to } 14 \\
15 \text { to } 21 \\
22 \text { onwards }\end{array}$ & $\begin{array}{l}39 \\
48 \\
38 \\
42\end{array}$ & $\begin{array}{l}44 \\
42 \\
39 \\
57\end{array}$ & $0 \cdot 36$ & $\begin{array}{l}62 \\
63 \\
48 \\
46\end{array}$ & $\begin{array}{l}60 \\
40 \\
52 \\
37\end{array}$ & 0.05 \\
\hline
\end{tabular}

study period, accounting for $26 \%$ of swabs taken. $U$ urealyticum was isolated during the first three weeks of the study; it was not isolated during the next three weeks but returned in weeks seven and eight. This pattern suggests that the negative swabs were not false negatives. This woman was asymptomatic throughout.

\section{Discussion}

Few longitudinal studies of the vaginal flora have been performed, and this is the most extensive that has been reported to date. In 1977 Bartlett performed quantitative bacteriology on samples collected from five volunteers at intervals throughout the menstrual cycle. ${ }^{5} \mathrm{He}$ found that the concentrations of anaerobes were maintained at relatively constant levels throughout the menstrual cycle, but that the concentrations of aerobic bacteria decreased in the premenstrual week. Analysis of sequential specimens showed considerable variation in the species recovered. In 1980 Sautter collected serial specimens from seven nurses during one menstrual cycle. ${ }^{6} \mathrm{He}$ too found a cyclical variation, with bacteroides being isolated mainly during the first half of the menstrual cycle. He also found $U$ urealyticum constantly in one, and intermittently in five, of the seven subjects. In 1982 Larsen cultured the vaginal fluid of 31 women during and after menstruation ${ }^{7} ; 19$ women showed a decrease in the number of anaerobes and 22 a decrease in the number of aerobes after menstruation.

In our study, $38 \%$ of healthy women had intermittent $\mathrm{BV}, 38 \%$ intermittent $U$ urealyticum, and $31 \%$ intermittent candidiasis; only $15 \%$ had apparently completely normal flora throughout. Many women had symptoms, but these were not closely related to the microbiological findings. Cultures for Neisseria gonorrhoeae and Chlamydia trachomatis were not performed as this would have compromised the anonymous nature of the study; 
however, vaginal discharge is an uncommon presentation of these infections, and they are unlikely to have been a common cause of the symptoms described. Because of potential delays in plating out the swabs, we placed more reliance on the microscopy than culture of the specimens, and did not perform quantitative cultures. However, microscopy of a Gram stained smear has been shown to be both sensitive and specific in the diagnosis of $\mathrm{BV},{ }^{8}$ and both our microscopy and culture results, using standard diagnostic methods widely used in genitourinary clinics, are in accordance with these previous studies in demonstrating the dynamic nature of the microbial population of the vaginal flora and its cyclical variation.

It has been recognised that BV and candidiasis may resolve spontaneously without treatment'; however, the high frequency with which these conditions may occur intermittently may not have been appreciated until now. We have demonstrated that abnormalities may occur for only a few consecutive days before resolving spontaneously. This has implications for future studies; if swabs are taken less frequently or indeed only on a single occasion, episodes of $\mathrm{BV}$ or candidiasis may be missed, or a single positive swab may be given more emphasis than it deserves. In common with other studies, ${ }^{10}$ we found BV more commonly in women having unprotected sex, but it was only found consistently in one woman who was not sexually active. This provides further evidence that $\mathrm{BV}$ is not necessarily sexually transmitted.

We had reservations about the accuracy of the $\mathrm{pH}$ reading from a blind vaginal swab, because of possible contamination from the vulva or cervical mucosa, and it was not measured by all the participants. However, the results in these women are presented because of their potential significance; it has not yet been established whether the rise in $\mathrm{pH}$ that is found in association with $\mathrm{BV}$ is a cause or an effect. Our finding that the change to the BV type flora is preceded by a rise in $\mathrm{pH}$ would, if confirmed, suggest that the $\mathrm{pH}$ increase may be a cause rather than an effect of the condition.

Our results are consistent with the theory that semen is one of a number of factors that can alter the environment of the vagina, possibly by raising the $\mathrm{pH}$, and trigger off a change in the flora. We looked at some of the external factors which could potentially alter the environment of the vagina. $\mathrm{BV}$ seemed to be related to frequent use of scented soap, and there appeared to be an additive effect of clothing and hygiene factors. Obviously no firm conclusions can be drawn in view of the small numbers studied, and in addition, the results may have been confounded by this highly aware group of women, especially those who have suffered from symptomatic candidiasis, avoiding the known or suspected precipitants. However, this study emphasises the multifactorial aetiology of BV, and we feel that these factors warrant further investigation.

The significance of $U$ urealyticum and $M_{\wp}$ hominis as pathogens has not been establishedD without doubt. U urealyticum is an infrequento cause of urethritis in men ${ }^{11}$ and it is suggested that it can be sexually transmitted. In our study its presence was not related to exposure to semen, and indeed it was found most fre quently in a woman who had never had sex? with a man. Similarly, the significance of the $\beta$-haemolytic streptococcus as a vaginal pathogen is unclear, although it has been associated with an adverse outcome of pregnancy. ${ }^{12}$ In our study it was only foundes sporadically in two women, both of whom also $\overrightarrow{0}$ had Candida spp., and it could not be determined whether it was a contaminant or a nor $-\stackrel{\omega}{-}$ mal constituent of the vaginal flora.

\section{Conclusion}

Our study raises doubts about what should be을 regarded as normal vaginal flora. It calls intoquestion the significance of finding bacteriald vaginosis, Candida spp., $M$ hominis, $U$ urealyticum, or $\beta$-haemolytic streptococci on a $\sin \frac{\Phi}{2}$ gle occasion in asymptomatic women, as well as the significance of finding normal flora on ac single occasion in symptomatic women.

We are grateful to our colleagues in Sheffield, Barnsley, and Rotherham who so readily participated in this study, and to Ms Pauline Whitaker for her help in preparing the manuscript.

1 Gardner HL, Dukes CD. Haemophilis vaginalis vaginitis.=

Am f Obstet Gynecol 1955;69:962-72.
Spiegel CA, Amsel R, Eschenbach D, Schoenkneckt R, Holmes KK. Anaerobic bacteria in non-specific vaginitis. $N$ Eng f Med 1980;303:601-7.

3 Spiegel CA, Davick P, Totten PA, Chen KCS, Eschenbach $\mathrm{DA}$, Amsel R, Holmes KK. Gardnerella vaginalis and anaerobic bacteria in the etiology of bacterial vaginosis. Scand $\mathcal{F}$ Inf Dis Suppl 1983;40:41-3.

4 Nugent RP, Krohn MA, Hillier SL. Reliability of diagnosing. bacterial vaginosis is improved by standard method of Gram stain interpretation. $\mathcal{F}$ Clin Microbiol 1991;29:297-301.

5 Bartlett JG, Onderdonk AB, Drude E, Goldstein C,O Anderka M, Alpert S, McCormack WM. Quantitative bacteriology of the vaginal flora. F Infect Dis 1977;136:O 271-7

6 Sautter RL, Brown WJ. Sequential vaginal cultures from normal young women. $\mathcal{f}$ Clin Microbiol 1980;11:479-84.

7 Larsen B, Galask RP. Vaginal microbial flora: composition and influences of host physiology. Ann Int Med 1982;96:응

8 Hay PE, Taylor-Robinson D, Lamont RF. The diagnosis of bacterial vaginosis in a gynaecology clinic. Br $\mathcal{F}$ Obstet

9 Moi H. Prevalence of bacterial vaginosis and its association with genital infections, inflammation, and contraceptive methods in women attending sexually transmitted disease and primary health clinics. Int $\mathcal{f}$ STD AIDS 1990;1:86-94.

10 Bowie WR. Urethritis in males. In: Holmes $\mathrm{KK}, \mathrm{Mardh}^{\mathbb{D}}$ PA, Sparling PF, Weisner PJ, eds. Sexually Transmitted

11 Janier M, Lassau F, Casin I, Grelot T, Scieux C, Zavavo A, et al. Male urethritis with and without discharge: a clinical and microbiological study. Sex Transm Dis 1995;22: and $\mathrm{mic}$. 52 .

12 Gibbs RS, Romero R, Hillier SL, Eschenbach DA, Sweet RL. A review of premature birth and subclinical infectê tion. Am f Obstet Gynecol 1992;166:1515-28. 